\title{
A Phase II study of the safety and efficacy of lenvatinib in patients with advanced thyroid cancer
}

\author{
Shunji Takahashi*,1, Naomi Kiyota², Tomoko Yamazaki ${ }^{3}$, Naoko Chayahara², Kenji \\ Nakano' ${ }^{1}$, Lina Inagaki ${ }^{1}$, Kazuhisa Toda ${ }^{4}$, Tomohiro Enokida ${ }^{5}$, Hironobu Minami²,

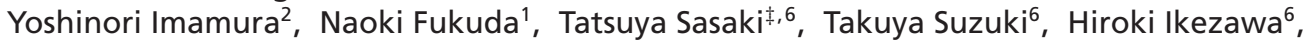 \\ Corina E Dutcus ${ }^{7}$ \& Makoto Tahara ${ }^{5}$ \\ ${ }^{1}$ Department of Medical Oncology, Cancer Institute Hospital of the Japanese Foundation for Cancer Research, Tokyo, Japan \\ ${ }^{2}$ Department of Medical Oncology \& Hematology/Cancer Center, Kobe University Hospital, Kobe, Japan \\ ${ }^{3}$ Department of Head \& Neck Oncology, Miyagi Cancer Center, Miyagi, Japan \\ ${ }^{4}$ Department of Head \& Neck Surgery, Cancer Institute Hospital of the Japanese Foundation for Cancer Research, Tokyo, Japan \\ ${ }^{5}$ Department of Head \& Neck Medical Oncology, National Cancer Center Hospital East, Kashiwa, Japan \\ ${ }^{6}$ Eisai Co. Ltd, Tokyo, Japan \\ ${ }^{7}$ Eisai Inc., Woodcliff Lake, NJ 07677, USA \\ *Author for correspondence: Tel.: +81 35200111 ext. 1474; Fax: +81 335700 343; s.takahashi-chemotherapy@jfcr.or.jp \\ †Former employee
}

Aim: To investigate the safety and efficacy of lenvatinib in advanced thyroid cancer. Patients/methods: In this Phase II study, 51 Japanese patients with radioiodine-refractory differentiated thyroid cancer (RR-DTC), medullary thyroid cancer (MTC) or anaplastic thyroid cancer (ATC) received once-daily lenvatinib $24 \mathrm{mg}$. The primary end point was safety. Results: All patients experienced $\geq 1$ adverse event (AE); only one patient experienced an $A E$ leading to discontinuation. The most common any-grade AEs were hypertension, decreased appetite, palmar-plantar erythrodysesthesia, fatigue and proteinuria. Response rates for RR-DTC: $68 \%$; MTC: $22 \%$; ATC: $24 \%$. Median progression-free survival for RR-DTC: 25.8 months; MTC: 9.2 months; ATC: 7.4 months. Conclusion: Lenvatinib demonstrated a manageable safety profile, proven antitumor activity in RR-DTC and promising efficacy in MTC and ATC.

Clinical trial registration: clinicaltrials.gov NCT01728623

Lay abstract: Lenvatinib is approved in Japan and elsewhere to treat radioiodine-refractory differentiated thyroid cancer; however, new therapies are also needed for other forms of advanced thyroid cancer (such as medullary thyroid cancer and anaplastic thyroid cancer). Fifty-one patients from Japan with advanced thyroid cancer received lenvatinib in this clinical trial. All patients experienced at least one side effect during treatment but only one patient experienced a side effect that necessitated stopping lenvatinib therapy. In general, patients tolerated lenvatinib treatment because the side effects were manageable. Lenvatinib treatment showed promising antitumor effects for patients across all thyroid cancer subtypes tested.

First draft submitted: 24 July 2018; Accepted for publication: 26 November 2018; Published online: 14 January 2019

Keywords: anaplastic $\bullet$ lenvatinib $\bullet$ medullary $\bullet$ radioiodine-refractory differentiated thyroid cancer $\bullet$ thyroid carcinoma

Thyroid cancer is clinically diverse, but can be grouped into three main subtypes: differentiated thyroid cancer (DTC; $94.3 \%$ of cases), medullary thyroid cancer (MTC; $2.2 \%$ of cases) and anaplastic thyroid cancer (ATC; $1.3 \%$ of cases) [1]. Initial treatment options for thyroid cancer are dependent on the type and the disease stage at diagnosis. DTC is initially treated with surgery, followed by radioactive iodine ablation therapy to reduce the risk of recurrence and metastatic disease [2]. Some patients (up to 15\%) will develop radioiodine-refractory DTC (RR-DTC) [3], which has a 10-year survival rate of $10 \%$ from time of detection of metastatic disease [4]. MTC, which usually occurs spontaneously but can be hereditary in up to $25 \%$ of cases [5], may be successfully treated with

Future $\because$ Medicine 
surgery in the first instance if diagnosed early when the disease is localized; however, once it metastasizes, survival time is generally short [5]. Nearly $50 \%$ of patients with ATC present with metastatic disease at initial diagnosis [6]. The current treatment options for ATC are limited, and the mortality rate is estimated at $90 \%$ in the first year $[7,8]$. New, effective, systemic therapies are, therefore, needed to treat all advanced thyroid cancer subtypes that have metastasized beyond a stage that can be treated with localized therapy or resection.

Lenvatinib is an oral multityrosine kinase inhibitor that acts on the VEGF receptors 1-3, FGF receptors 1-4, PDGF receptor- $\alpha$ and RET and KIT proto-oncogenes [9-11]. Lenvatinib was approved for the treatment of RR-DTC based on the pivotal Phase III Study of (E7080) Lenvatinib in Differentiated Cancer of the Thyroid (SELECT), a randomized, double-blind placebo-controlled trial in which lenvatinib significantly prolonged progression-free survival (PFS) in patients with RR-DTC compared with placebo (median PFS: 18.3 vs 3.6 months, respectively; $\mathrm{p}<0.001)$ [12]. SELECT included an exploratory analysis of potential biomarkers associated with clinical outcomes; however, this analysis was limited to circulating cytokine/angiogenic factors and mutations of the BRAF, NRAS, KRAS and HRAS genes [13]. Recently, the role of the neutrophil-to-lymphocyte ratio (NLR) - a measure of inflammation - as a biomarker for survival in several types of cancers has attracted more attention [14-16].

This Phase II study was undertaken primarily to assess the safety of once-daily lenvatinib in predominantly Japanese patients with advanced thyroid cancers (including RR-DTC, MTC, and ATC). An exploratory analysis of the NLR and its association with clinical outcome was also performed.

\section{Patients \& methods}

Patients

Patients aged 20 years or older were eligible if they had Eastern Cooperative Oncology Group (ECOG) performance status of 0-2; systolic blood pressure $\leq 140 \mathrm{mmHg}$ and diastolic blood pressure $\leq 90 \mathrm{mmHg}$; adequate renal, bone marrow, liver and blood coagulation functions; and histologically or cytologically confirmed diagnosis of advanced thyroid cancer of any of the specified subtypes (RR-DTC, MTC or ATC).

Patients with RR-DTC must have had disease progression (according to Response Evaluation Criteria in Solid Tumors version 1.1 [RECIST v1.1], confirmed by computerized tomography [CT] or MRI within 12 months before informed consent), a measurable lesion, been receiving thyroxine suppression therapy, and been confirmed as radioiodine-refractory or resistant (one or more measurable lesions with no iodine uptake per radioiodine scanning, or one or more measurable lesions that progressed per RECIST criteria within 12 months of radioiodine therapy despite uptake, or cumulative activity of radioiodine of $>600 \mathrm{mCi}$ or 22 gigabecquerel, with the last dose administered at least 6 months before study entry).

Patients with MTC were required to have histologically or cytologically confirmed diagnosis of MTC, evidence of disease progression according to RECIST v1.1 (by CT or MRI) within 12 months before informed consent, and/or clinical disease progression, and an evaluable target lesion or nontarget lesion according to RECIST v1.1.

Patients with ATC were required to have had an evaluable target lesion according to RECIST v1.1, agreed to hospitalization in cycle 1 , and be expected to survive for 8 weeks or longer after the first dose of the study drug. Key exclusion criteria included concomitant brain metastases (unless previously treated and clinically stable for $\geq 1$ month prior to screening), history of cardiovascular impairment, bleeding, or thrombotic disorders, or use of anticoagulants such as warfarin, proteinuria or previous treatment with lenvatinib.

\section{Study design}

This was a nonrandomized, open-label, multicenter, Phase II study. The trial was conducted at three study sites in Japan from 3 September 2012 to 9 July 2015. Study enrollment continued until lenvatinib was approved for unresectable thyroid cancer in Japan. After approval, the trial continued as a postmarketing study until lenvatinib was commercially available at each site. The protocol was amended to reflect this, but the end points and analyses were not changed.

Patients received a once-daily oral lenvatinib dose of $24 \mathrm{mg}$ in 28-day continuous cycles. Treatment continued until disease progression according to RECIST v1.1 or development of unacceptable toxicity occurred. For patients with RR-DTC and MTC, tumor assessments were performed every 8 weeks after the first dose based on investigator assessment per RECIST v1.1. For patients with ATC, tumor assessments were performed at 4, 8, 12 and 16 weeks after the first dose, and every 8 weeks thereafter, based on investigator assessment per RECIST v1.1.

The study was designed in accordance with the Guidelines for Clinical Evaluation of Anti-Cancer Drugs in Japan and with the Declaration of Helsinki and local laws. All patients provided written informed consent. 
The institutional review boards of each medical institution (National Cancer Center Hospital East, Kashiwa, Japan; the Cancer Institute Hospital of Japanese Foundation for Cancer Research, Tokyo, Japan; and the Kobe University Hospital, Kobe, Japan) approved the study and protocol. The trial was registered at www.clinicaltrials.gov (NCT01728623). Statistical analyses were performed by Eisai Co., Ltd, and Takumi Information Technology Inc.

\section{Safety \& efficacy analyses}

The primary objective was to evaluate the safety of lenvatinib in patients with advanced thyroid cancer. Safety measures included recording of adverse events (AEs), clinical laboratory results (including urinalysis, hematology and blood chemistry), vital signs, weight, electrocardiography and physical exam. AEs were graded using Common Terminology Criteria for AEs version 4.0 (CTCAE v4.0).

Efficacy measures were secondary end points. Key efficacy analyses included PFS (defined as time from the date of first dose to first documentation of disease progression as determined by investigator assessment or death from any causes, whichever occurred first), overall survival (OS; defined as time from the date of first dose to the date of death from any cause), objective response rate (defined as the proportion of patients with a complete or partial response [PR] by RECIST v1.1), disease control rate (defined as the proportion of patients with complete or PR, or stable disease) and clinical benefit rate (defined as the proportion of patients with complete or PR, or durable stable disease [defined as $\geq 23$ weeks for DTC and MTC; $\geq 11$ weeks for ATC]). An exploratory analysis was undertaken to investigate the NLR at baseline and its effect on PFS.

\section{Statistical analysis}

Statistical analyses were performed using Statistical Analysis System (version 9.2 or later). The sample size was based on feasibility. A sample size of $\geq 16$ was planned, which was estimated to have a probability of $>0.8$ (or $80 \%$ ) to detect AEs with a frequency of $\geq 10 \%$. No adjustments for covariates and no statistical comparisons were planned. PFS and OS outcomes were summarized using Kaplan-Meier estimates. For the exploratory biomarker analysis, the effects of baseline NLR on PFS were evaluated using a Cox proportional hazard model. Each cancer classification had varied baseline NLR and PFS results, and were therefore also included in the model as a covariate, for adjustment purposes.

\section{Results}

Results for the ATC subgroup in this study have been previously reported [17]. Here, we provide the overall results of the study, inclusive of all thyroid cancer subtypes.

\section{Disposition}

The disposition of the study patients has been previously published [17]. In brief, between 3 September 2012 and 9 July 2015, 60 patients were screened for the study, and 51 (RR-DTC, $\mathrm{n}=25$; MTC, $\mathrm{n}=9$; ATC, $\mathrm{n}=17$ ) were enrolled. All 51 patients received at least one dose of the study drug. Twenty-three patients discontinued due to the termination of the study after approval of lenvatinib, and a further 26 discontinued the study drug due to disease progression. One patient discontinued the study-drug treatment due to a treatment-emergent AE (TEAE), and one discontinued the study drug by choice. All 51 patients were included in the efficacy and safety analysis sets.

\section{Baseline demographics \& characteristics}

Most (59\%) patients were women, and all patients were Japanese. Baseline patient demographics and disease characteristics are shown in Table 1. Baseline ECOG scores were either 0 or 1 for most patients, except for two patients from the ATC group who had an ECOG score of 2 [17]. Surgery was the most common previous treatment across all three thyroid cancer types. Other prior treatments received are listed in Table 1. Of note, all patients with RR-DTC in the study had received previous radioiodine therapy, and only three patients (RR-DTC, $\mathrm{n}=2$; MTC, $\mathrm{n}=1$ ) had received prior treatment with a VEGF-targeted agent for their cancer. As previously reported, chemotherapy had mostly been received by patients with ATC [17].

\section{Exposure to study drug}

Overall, the median number of cycles of study drug received was 15.0 (range: 1-37), and the median duration of exposure was 407 days (range: 22-1009), or 13.4 months (range: 0.7-33.1). In patients with RR-DTC, the median duration of exposure was 727 days (range: 22-981) or 23.9 months (range: 0.7-32.2). In patients with 


\begin{tabular}{|c|c|c|c|c|}
\hline Parameter & RR-DTC $(n=25)$ & $\operatorname{MTC}(n=9)$ & $\operatorname{ATC}(n=17)$ & Overall $(\mathrm{N}=51)$ \\
\hline Age, median (range), years & $58(21-74)$ & $58(41-79)$ & $65(36-84)$ & $61(21-84)$ \\
\hline Female sex, n (\%) & $16(64)$ & $3(33)$ & $11(65)$ & $30(59)$ \\
\hline Weight, median (range), kg & $57(44-99)$ & $52(40-78)$ & $54(40-85)$ & $56(40-99)$ \\
\hline \multicolumn{5}{|l|}{ ECOG performance status, $\mathrm{n}(\%)$ : } \\
\hline-0 & $17(68)$ & $8(89)$ & $5(29)$ & $30(59)$ \\
\hline-1 & $8(32)$ & $1(11)$ & $10(59)$ & $19(37)$ \\
\hline-2 & 0 & 0 & $2(12)$ & $2(4)$ \\
\hline Baseline lesion diameter, median (range), $\mathrm{mm}$ & $51(19-217)$ & $56(20-202)$ & $52(15-232)$ & - \\
\hline \multicolumn{5}{|l|}{ Previous anticancer therapy or therapies, $\mathrm{n}(\%)$ : } \\
\hline - Surgery & $25(100)$ & $8(89)$ & $14(82)$ & $47(92)$ \\
\hline - Chemotherapy & $1(4)$ & 0 & $7(41)$ & $8(16)$ \\
\hline - VEGF therapy & $2(8)$ & $1(11)$ & 0 & $3(6)$ \\
\hline - Radiotherapy & $8(32)$ & $1(11)$ & $9(53)$ & $18(35)$ \\
\hline
\end{tabular}

\begin{tabular}{|c|c|c|c|c|}
\hline Category & $\begin{array}{l}\text { RR-DTC } \\
(n=25) \\
n(\%)\end{array}$ & $\begin{array}{l}\text { MTC } \\
(n=9) \\
n(\%)\end{array}$ & $\begin{array}{l}\text { ATC } \\
(n=17) \\
n(\%)\end{array}$ & $\begin{array}{l}\text { Overall } \\
(\mathrm{N}=51) \\
\mathrm{n}(\%)\end{array}$ \\
\hline TEAEs: & $25(100)$ & $9(100)$ & $17(100)$ & $51(100)$ \\
\hline - Treatment-related TEAEs & $25(100)$ & $9(100)$ & $17(100)$ & $51(100)$ \\
\hline - CTCAE Grade 3 or 4 TEAEs & $18(72)$ & $9(100)$ & $15(88)$ & $42(82)$ \\
\hline SAEs: & $8(32)$ & $6(67)$ & $13(77)$ & $27(53)$ \\
\hline - Deaths & 0 & $1(11)$ & $3(18)$ & $4(8)^{\dagger}$ \\
\hline \multicolumn{5}{|l|}{ - Other SAEs: } \\
\hline - Life threatening & 0 & 0 & $1(6)$ & $1(2)$ \\
\hline - Required inpatient hospitalization or prolongation of existing hospitalization & $7(28)$ & $6(67)$ & $13(77)$ & $26(51)$ \\
\hline \multicolumn{5}{|l|}{ TEAEs leading to study-drug dose adjustment: } \\
\hline - TEAEs leading to study-drug withdrawal & 0 & 0 & $1(6)$ & $1(2)$ \\
\hline - TEAEs leading to study-drug dose reduction & $25(100)$ & $9(100)$ & $15(88)$ & $49(96)$ \\
\hline - TEAEs leading to study-drug interruption & $17(68)$ & $6(67)$ & $11(65)$ & $34(67)$ \\
\hline \multicolumn{5}{|c|}{$\begin{array}{l}\text { † Occurring within } 30 \text { days of the last dose of study drug. } \\
\text { A patient with two or more TEAEs in that category is counted only once; if TEAEs had different CTCAE grades, then the event with the highest grade was used for that patient. } \\
\text { Treatment-related TEAEs were considered by the investigator to be possibly or probably related to study drug. } \\
\text { ATC: Anaplastic thyroid cancer; CTCAE: Common Terminology Criteria for Adverse Event; MTC: Medullary thyroid cancer; RR-DTC: Radioiodine-refractory differentiated thyroid } \\
\text { cancer; SAE: Serious adverse event; TEAE: Treatment-emergent adverse event. }\end{array}$} \\
\hline
\end{tabular}

MTC, median duration of exposure was 293 days (range: 77-867) or 9.6 months (range: 2.5-28.5); for patients with ATC, 166 days (range: 22-1009) or 5.5 months (range: 0.7-33.1). Overall, the median percentage of received dose versus planned starting dose (24 mg/d) was 47\% (range: $21-100 \%)$ (Supplemental Table 1).

Safety

Table 2 presents an overview of TEAEs. All 51 (100\%) patients experienced at least one TEAE, and all patients also had at least one treatment-related TEAE. Most $(\mathrm{n}=42 ; 82 \%)$ patients had at least one CTCAE grade 3 or 4 TEAE. Serious AEs (SAEs) occurred in 27 (53\%) patients. Five patients experienced a fatal SAE, four of whom died within 30 days of the last dose of study drug. All fatal SAEs were reported as unrelated to the study drug by the investigator. One death was due to hepatic failure caused by liver metastases associated with disease progression, and the other four deaths were due to disease progression.

A TEAE of suicide attempt led to study-drug withdrawal in one (2\%; with ATC) patient. TEAEs leading to study-drug dose reduction occurred in $49(96 \%)$ patients. All TEAEs leading to lenvatinib-dose reduction were 


\begin{tabular}{|c|c|c|c|c|}
\hline Preferred MedDRA Term, $\mathrm{n}(\%)$ & RR-DTC $(n=25)$ & $\operatorname{MTC}(n=9)$ & ATC $(n=17)$ & Overall $(\mathrm{N}=51)$ \\
\hline Patients with any TEAE & $25(100)$ & $9(100)$ & $17(100)$ & $51(100)$ \\
\hline Hypertension & $24(96)$ & $8(89)$ & $14(82)$ & $46(90)$ \\
\hline Decreased appetite & $17(68)$ & $9(100)$ & $14(82)$ & $40(78)$ \\
\hline Palmar-plantar erythrodysesthesia syndrome & $23(92)$ & $8(89)$ & $8(47)$ & $39(77)$ \\
\hline Fatigue & $20(80)$ & $7(78)$ & $10(59)$ & $37(73)$ \\
\hline Proteinuria & $15(60)$ & $6(67)$ & $10(59)$ & $31(61)$ \\
\hline Stomatitis & $17(68)$ & $4(44)$ & $8(47)$ & $29(57)$ \\
\hline Diarrhea & $15(60)$ & $8(89)$ & $5(29)$ & $28(55)$ \\
\hline Dysphonia & $10(40)$ & $4(44)$ & $7(41)$ & $21(41)$ \\
\hline Nausea & $9(36)$ & $2(22)$ & $10(59)$ & $21(41)$ \\
\hline Arthralgia & $14(56)$ & $1(11)$ & $4(24)$ & $19(37)$ \\
\hline Weight decreased & $5(20)$ & $4(44)$ & $7(41)$ & $16(31)$ \\
\hline Nasopharyngitis & $11(44)$ & $1(11)$ & $2(12)$ & $14(28)$ \\
\hline Edema peripheral & $5(20)$ & $4(44)$ & $5(29)$ & $14(28)$ \\
\hline Constipation & $3(12)$ & $3(33)$ & $7(41)$ & $13(26)$ \\
\hline Headache & $5(20)$ & $3(33)$ & $5(29)$ & $13(26)$ \\
\hline Abdominal pain upper & $5(20)$ & $4(44)$ & $3(18)$ & $12(24)$ \\
\hline Myalgia & $8(32)$ & $2(22)$ & $2(12)$ & $12(24)$ \\
\hline Vomiting & $4(16)$ & $2(22)$ & $6(35)$ & $12(24)$ \\
\hline Thrombocytopenia & $4(16)$ & $2(22)$ & $5(29)$ & $11(22)$ \\
\hline Alopecia & $7(28)$ & 0 & $3(18)$ & $10(20)$ \\
\hline Epistaxis & $4(16)$ & $2(22)$ & $4(24)$ & $10(20)$ \\
\hline Rash & $6(24)$ & $2(22)$ & $2(12)$ & $10(20)$ \\
\hline Dermatitis acneiform & $6(24)$ & 0 & $3(18)$ & $9(18)$ \\
\hline Insomnia & 1 (4) & $5(56)$ & $3(18)$ & $9(18)$ \\
\hline Cough & $5(20)$ & 0 & $3(18)$ & $8(16)$ \\
\hline Cancer pain & $3(12)$ & $2(22)$ & $2(12)$ & $7(14)$ \\
\hline Hypocalcemia & $3(12)$ & $2(22)$ & $2(12)$ & $7(14)$ \\
\hline Hypothyroidism & 0 & $3(33)$ & $4(24)$ & $7(14)$ \\
\hline Malaise & 1 (4) & $3(33)$ & $3(18)$ & $7(14)$ \\
\hline Aspartate aminotransferase increased & 0 & $1(11)$ & $5(29)$ & $6(12)$ \\
\hline Back pain & $4(16)$ & $1(11)$ & $1(6)$ & $6(12)$ \\
\hline Dehydration & $2(8)$ & 0 & $4(24)$ & $6(12)$ \\
\hline Hepatic function abnormal & $2(8)$ & $2(22)$ & $2(12)$ & $6(12)$ \\
\hline Pneumonia & $3(12)$ & 0 & $3(18)$ & $6(12)$ \\
\hline Pyrexia & $1(4)$ & $1(11)$ & $4(24)$ & $6(12)$ \\
\hline
\end{tabular}

grade 2 or grade 3 , except for two events (grade 4 thrombocytopenia and grade 4 hypocalcemia). TEAEs leading to study-drug interruption occurred in 34 (67\%) patients.

Overall, the most frequently reported TEAE was hypertension $(n=46 ; 90 \%)$, followed by decreased appetite, palmar-plantar erythrodysesthesia, fatigue, proteinuria, stomatitis, diarrhea, dysphonia and nausea (Table 3). Of the 40 patients who experienced decreased appetite, 14 also had decreased weight as a TEAE. The most frequently reported treatment-related TEAE was hypertension (90\%), followed by palmar-plantar erythrodysesthesia (75\%), decreased appetite (73\%), fatigue (69\%), proteinuria (61\%), stomatitis $(57 \%)$ and diarrhea $(53 \%)$.

Grade 3 or 4 TEAEs were experienced by $42(82 \%)$ patients. The most frequently reported grade 3 or grade 4 TEAE was hypertension in 22 patients (43\%; RR-DTC, $n=15$; MTC, $n=2$; ATC, $n=5$ ), followed by decreased appetite in six patients (12\%; RR-DTC, $n=1$; MTC, $n=2$; ATC, $n=3)$. Four patients experienced one or more 
Table 4. Summary of tumor response.

\begin{tabular}{|c|c|c|c|c|}
\hline Parameter & RR-DTC $(n=25)$ & MTC $(n=9)$ & $\operatorname{ATC}(n=17)$ & Overall $(\mathrm{N}=51)$ \\
\hline \multicolumn{5}{|l|}{ Best overall response: } \\
\hline$-C R, n$ & 0 & 0 & 0 & 0 \\
\hline - PR, n (\%) & $17(68)$ & $2(22)$ & $4(24)$ & $23(45)$ \\
\hline$-\mathrm{SD}^{\dagger}, \mathrm{n}(\%)$ & $8(32)$ & $7(78)$ & $12(71)$ & $27(53)$ \\
\hline - PD, n (\%) & 0 & 0 & $1(6)$ & $1(2)$ \\
\hline$-N E, n$ & 0 & 0 & 0 & 0 \\
\hline ORR (CR+PR), n (\%) & $17(68)$ & $2(22)$ & $4(24)$ & $23(45)$ \\
\hline $\mathrm{DCR}(\mathrm{CR}+\mathrm{PR}+\mathrm{SD}), \mathrm{n}(\%)$ & $25(100)$ & $9(100)$ & $16(94)$ & $50(98)$ \\
\hline $\mathrm{CBR}\left(\mathrm{CR}+\mathrm{PR}+\mathrm{dSD} \mathrm{D}^{\ddagger}\right), \mathrm{n}(\%)$ & $21(84)$ & $7(78)$ & $12(71)$ & $40(78)$ \\
\hline \multicolumn{5}{|c|}{$\begin{array}{l}\text { The tumor assessment was evaluated based on RECIST v1.1 criteria by the investigator. } \\
\text { †SD is defined as SD lasting } \geq 7 \text { weeks for DTC and MTC, and } \geq 3 \text { weeks for ATC. } \\
\text { ¥dSD is defined as SD lasting } \geq 23 \text { weeks for DTC and MTC, and } \geq 11 \text { weeks for ATC. } \\
\text { ATC: Anaplastic thyroid cancer; CBR: Clinical benefit rate; CR: Complete response; DCR: Disease control rate; dSD: Durable stable disease; MTC: Medullary thyroid cancer; ORR: Objective } \\
\text { response rate; PD: Progressive disease; PR: Partial response; RECIST v1.1: Response Evaluation Criteria in Solid Tumors version 1.1; RR-DTC: Radioiodine-refractory differentiated thyroid } \\
\text { cancer; SD: Stable disease. }\end{array}$} \\
\hline
\end{tabular}

grade 4 TEAEs (hypocalcemia, hyponatremia, laryngeal stenosis, suicide attempt and thrombocytopenia). The grade 4 hypocalcemia and thrombocytopenia events were reported as treatment related by the investigator. The grade 4 TEAEs were resolved with treatment or study-drug dose reduction, with the exception of hypocalcemia, laryngeal stenosis and suicide attempt.

\section{Efficacy}

The median PFS in patients with RR-DTC was 25.8 months (95\% CI: 18.4-not reached [NR]); in patients with MTC, 9.2 months (95\% CI: 1.8-NR); and in patients with ATC, 7.4 months (95\% CI: 1.7-12.9). Median OS in patients with RR-DTC was 31.8 months (95\% CI: 31.8-NR); in patients with MTC, 12.1 months (95\% CI: 3.8-NR); and in patients with ATC, 10.6 months (95\% CI: 3.8-19.8).

Responses based on the investigator assessment are summarized in Table 4 . The objective response rate was $68 \%$ $(\mathrm{n}=17)$ in patients with RR-DTC, $22 \%(\mathrm{n}=2)$ in patients with MTC, and $24 \%(\mathrm{n}=4)$ in patients with ATC. No subgroup had a complete response. The disease control rate was $100 \%$ both in patients with RR-DTC $(\mathrm{n}=25)$ and with MTC $(\mathrm{n}=9)$ and $94 \%(\mathrm{n}=16)$ in patients with ATC. The clinical benefit rate was $84 \%(\mathrm{n}=21)$ in patients with RR-DTC, 78\% $(\mathrm{n}=7)$ in patients with MTC and 71\% $(\mathrm{n}=12)$ in patients with ATC. Most patients experienced some degree of tumor shrinkage after beginning study treatment (Figure 1). Of particular note, tumor samples from ten patients with ATC were sent for independent pathological review and all were confirmed as ATC; three of those ten patients achieved PR.

Neutrophil-to-lymphocyte ratio

At baseline, the overall mean NLR was 4.57 (standard deviation [SD]: \pm 5.06 ) and the median was 2.95 (range: $0.95-30.46$ ). For patients with RR-DTC, the mean baseline NLR was 2.82 (SD: \pm 1.43 ), and median was 2.30 (range: 0.95-6.19). In contrast, higher baseline NLRs were observed in patients with MTC (mean: 5.69 [SD: \pm 5.05 ]; median: 3.78 [range: 1.94-14.90]) and ATC (mean: 6.55 [SD: \pm 7.37 ]; median 4.38 [range: 1.41-30.46]).

A multivariable Cox proportional hazard model, with baseline NLR (as a continuous variable) and tumor subtypes (RR-DTC, MTC, ATC) as covariates, was used to estimate the hazard ratio for effect of NLR on PFS (Table 5). After adjustment by tumor subtype, a trend indicating that baseline NLR may affect PFS was seen, although significance was not reached in this exploratory analysis $(p=0.06)$. An additional multivariate analysis with NLR treated as a categorical variable (dichotomized as NLR $\geq 3$ vs $<3$, because median baseline NLR was 2.95) also showed the same nonsignificant trend (Supplemental Table 2; Supplemental Figure 1).

\section{Discussion}

This study demonstrated that lenvatinib had a manageable safety profile in patients with advanced thyroid cancer, irrespective of cancer subtype. The safety profile of lenvatinib in this Phase II study was comparable to that observed in other trials of lenvatinib in thyroid cancer [12,18-20]. The most common AEs were those known to be typical class effects of tyrosine kinase inhibitors, including hypertension, decreased appetite, palmar-plantar erythrodysesthesia, 


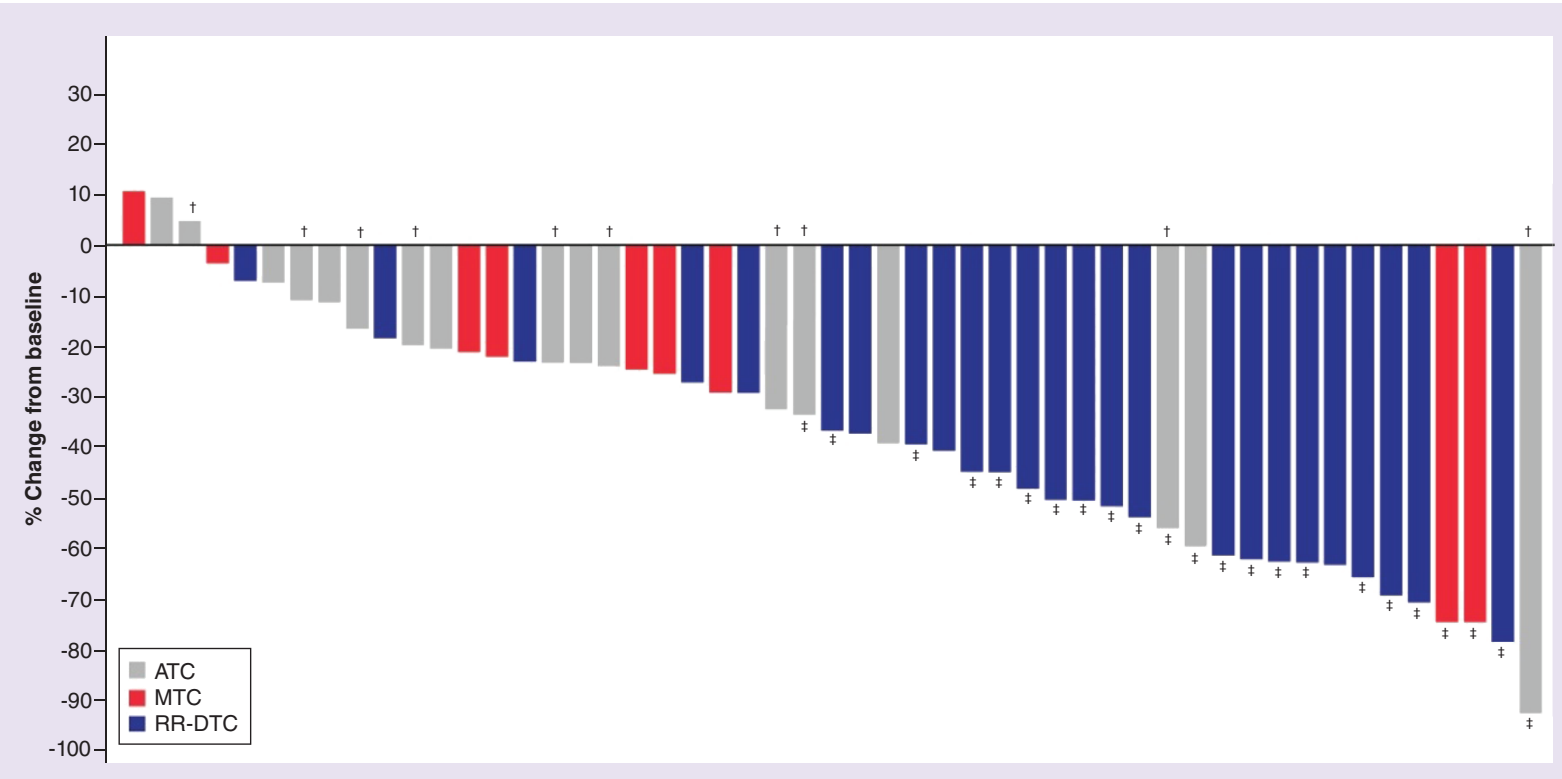

Figure 1. Maximum percentage change from baseline in the sum of diameters of target lesions.

${ }^{\dagger}$ ATC confirmed by independent pathologic review.

¥Patients with partial response as best overall response.

ATC: Anaplastic thyroid cancer; MTC: Medullary thyroid cancer; RR-DTC: Radioiodine-refractory differentiated thyroid cancer.

\begin{tabular}{|c|c|c|c|}
\hline Parameter & Hazard ratio & $95 \% \mathrm{Cl}$ & p-value \\
\hline MTC vs RR-DTC ${ }^{\dagger}$ & 2.32 & $0.83-6.52$ & 0.11 \\
\hline ATC vs RR-DTC ${ }^{\dagger}$ & 3.08 & $1.25-7.58$ & 0.01 \\
\hline
\end{tabular}

†Used as reference level in the model.

ATC: Anaplastic thyroid cancer; RR-DTC: Radioiodine-refractory differentiated thyroid cancer; MTC: Medullary thyroid cancer; NLR: Neutrophil-to-lymphocyte ratio.

fatigue, proteinuria, stomatitis and diarrhea $[18,21,22]$. Generally, toxicities were managed with dose interruptions and reductions, and only one patient (with ATC) withdrew from the study due to an AE.

Survival and response rates were highest in RR-DTC, followed by MTC and ATC. The efficacy of lenvatinib in RR-DTC has been demonstrated in the large-scale, placebo-controlled, Phase III SELECT in patients with RR-DTC, and held true irrespective of the criteria used to define RR-DTC [12,23]. The RR-DTC response rate in this study was also similar to that observed in SELECT, including in the subanalysis of Japanese patients who participated in that trial $[12,18]$.

In a Phase II study of sorafenib in patients with MTC or ATC in Japan, median PFS was not reached for MTC, but was 2.8 months for ATC, underscoring the difficult-to-treat nature of this disease [24]. The most common sorafenib-related any-grade $\mathrm{AE}$ and grade $\geq 3 \mathrm{AE}$ in that study was palmar-plantar erythrodysesthesia. In this current study of lenvatinib in patients with advanced thyroid cancer, hypertension was the most common anygrade and grade $\geq 3 \mathrm{AE}$. Although limited by indirect comparison and small sample sizes, results of these studies indicate some differences in the safety profiles of these two agents that are both used to treat advanced thyroid cancer in Japan.

NLR levels have been reported to be a prognostic marker for survival outcomes in several cancers [14-16], and higher NLR levels have been observed in more aggressive papillary thyroid carcinoma tumors [25,26]. Studies have also shown that NLR levels may reflect the pathological inflammatory status of patients with thyroid cancer [27,28]. Although not statistically significant, a trend was observed in this study indicating that increased baseline NLR might correlate with shorter PFS, regardless of thyroid cancer subtype. 
Compared with historical data reporting a median baseline NLR of 1.57 among 3870 patients with thyroid cancer [29], the baseline NLRs in this study were relatively high in all subtypes. This may indicate that the patients enrolled in our trial were experiencing aggressive disease, and were thus appropriately administered an anticancer therapy (in this case, lenvatinib). As of this writing, the optimal time to initiate lenvatinib treatment is unclear, with some physicians using disease progression within 6 months or thyroglobulin doubling time within 1 year for guidance [30]. Based on the preliminary analysis in this study, we hypothesize that the baseline NLR may be useful in providing an additional indicator for lenvatinib treatment initiation and should be investigated further. We acknowledge these inferences are limited by the nonrandomized trial design of the current study, the lack of a comparator arm, a small patient population and the lack of biomarker evaluation of tumor samples.

In conclusion, this study provides evidence supporting the use of lenvatinib in Japanese patients with advanced thyroid cancer, as lenvatinib demonstrated a manageable toxicity profile and promising antitumor activity across all thyroid cancer subtypes tested. In addition, results from an exploratory analysis suggested that the NLR may potentially be a prognostic marker for PFS and may possibly also be used as an indicator for timely initiation of lenvatinib treatment, pending further study.

\section{Future perspective}

In the upcoming years, additional therapies, both molecular-targeted and combination regimens, will likely be developed for the treatment of advanced thyroid cancer. The combination of a tyrosine kinase inhibitor with an immuno-oncology therapy has shown promise in other cancers, and may also be efficacious in patients with advanced thyroid cancer. Thus, with the number of potential therapies on the horizon, it is becoming increasingly important to determine which patients will derive the most benefit from a specific therapy. Future research into the molecular mechanisms of therapies, as well as the identification of biomarkers or predictors of response, will likely aid physicians in the process of selecting the best therapy for their patients.

\section{Summary points}

- Lenvatinib had a manageable safety profile in patients with advanced thyroid cancer $(\mathrm{N}=51)$, including radioiodine-refractory differentiated thyroid cancer (RR-DTC), medullary thyroid cancer (MTC) and anaplastic thyroid cancer (ATC).

- The safety profile of lenvatinib in this study was consistent with that seen in other clinical trials of lenvatinib, and was consistent with known class effects of tyrosine kinase inhibitors.

- Response rates were $68 \%$ for patients with RR-DTC, $22 \%$ for patients with MTC and $24 \%$ for patients with ATC.

- The median progression-free survival for patients with RR-DTC was 25.8 months; for patients with MTC, 9.2 months; and for patients with ATC, 7.4 months.

- The median overall survival for patients with RR-DTC was 31.8 months; for patients with MTC, 12.1 months; and for patients with ATC, 10.6 months.

- In a preliminary analysis, patients in this study were found to have had higher-than-expected neutrophil-tolymphocyte ratios, which may be indicative of aggressive disease.

\section{Supplementary data}

To view the supplementary data that accompany this paper please visit the journal website at: https://www.futuremedicine.com/d oi/suppl/10.2217/fon-2018-0557

\footnotetext{
Authors' contributions

S Takahashi performed study design, data collection, data interpretation, writing and reviewing manuscript. N Kiyota participated as a primary investigator, advised on the conducting of the study and enrolling of patients and also participated in the writing and reviewing of this manuscript. T Yamazaki, K Nakano, L Inagaki, K Toda, H Minami, T Sasaki and CE Dutcus performed data collection, data interpretation; and review of the manuscript. N Chayahara, Y Imamura and N Fukuda participated in patient management and review of the manuscript. T Enokida participated in patient management, and read and approved the final manuscript. T Suzuki performed study design, writing and review of the manuscript. H lkezawa performed data and statistical analyses and review of manuscript drafts. M Tahara performed data collection, data interpretation and review of the manuscript.
} 
Financial \& competing interests disclosure

This work was supported by Eisai Inc., USA. The authors declare the following relevant financial relationships: S Takahashi received grants and personal fees from Eisai (during the conduct of the study); grants and personal fees from Eisai, Novartis, Taiho, MSD, Chugai, Daiichi-Sankyo, Bayer and AstraZeneca. N Kiyota received grants for research funding from Eisai during the conduct of this study; and grants for research funding from Ono Pharmaceutical Co., Ltd, AstraZeneca Co., Ltd, and Pfizer, outside the submitted work; honoraria from Ono Pharmaceutical Co., Ltd, Bristol-Myers Squibb, Merck Serono, Eisai and Bayer. T Yamazaki, N Chayahara, K Nakano, L Inagaki, K Toda, T Enokida, Y Imamura and N Fukuda declared no conflicts of interest. H Minami received grants and personal fees from Eisai (during the conduct of the study); grants from Asahi-Kasei Pharma, Astellas, Nihon Shinyaku, TaishoToyama, Teijin Pharma, Yakult, Boehringer, Dainippon Sumitomo, Eisai, Kyowa-Kirin, Lilly, Nippon Chemiphar, Pfizer, Sanofi and Takeda; grants, personal fees, and other from Bayer, Bristol-Myers Squibb, Chugai, DaiichiSankyo, MSD, Novartis, Ono Yakuhin and Taiho; personal fees from Celgene, Janssen, Kowa, Merck Serono, Mochida, Otsuka, and Shire Japan; other from AstraZeneca; and consulting or advisory role with Merck Serono and Ono Pharmaceutical. M Tahara received grants and personal fees from Eisai (during the conduct of the study); grants and personal fees from AstraZeneca, Pfizer, Merck Sharp \& Dohme, Ono Pharmaceutical and Bristol-Myers Squibb. Personal fees from Bayer and Otsuka; grants from Novartis, NanoCarrier and Boehringer-Ingelheim. T Sasaki, T Suzuki, H Ikezawa and CE Dutcus are employees of Eisai (or were at the time of the study). The authors have no other relevant affiliations or financial involvement with any organization or entity with a financial interest in or financial conflict with the subject matter or materials discussed in the manuscript apart from those disclosed.

Medical writing support was provided by S Selamat, Oxford PharmaGenesis Inc., Newtown, PA, USA, and was funded by Eisai Inc.

\section{Ethical disclosure}

The authors state that they have obtained appropriate institutional review board approval or have followed the principles outlined in the Declaration of Helsinki for all human or animal experimental investigations. In addition, for investigations involving human subjects, informed consent has been obtained from the participants involved.

\section{Open access}

This work is licensed under the Attribution-NonCommercial-NoDerivatives 4.0 Unported License. To view a copy of this license, visit http://creativecommons.org/licenses/by-nc-nd/4.0/

\section{References}

Papers of special note have been highlighted as: $\bullet$ of interest; $\bullet \bullet$ of considerable interest

1. Aschebrook-Kilfoy B, Ward MH, Sabra MM, Devesa SS. Thyroid cancer incidence patterns in the United States by histologic type, 1992-2006. Thyroid 21(2), 125-134 (2011).

2. National Comprehensive Cancer Network Clinical Practice Guidelines in Oncology (NCCN Guidelines ${ }^{\circledR}$ ). Thyroid Carcinoma. Version 2.2017. www.nccn.org/professionals/physician_gls/pdf/thyroid.pdf

3. Pacini F, Ito Y, Luster M, Pitoia F, Robinson B, Wirth L. Radioactive iodine-refractory differentiated thyroid cancer: unmet needs and future directions. Expert Rev. Endocrinol. Metab. 7(5), 541-554 (2012).

4. Durante C, Haddy N, Baudin E et al. Long-term outcome of 444 patients with distant metastases from papillary and follicular thyroid carcinoma: benefits and limits of radioiodine therapy. J. Clin. Endocrinol. Metab. 91(8), 2892-2899 (2006).

5. Elisei R, Schlumberger MJ, Müller SP et al. Cabozantinib in progressive medullary thyroid cancer. J. Clin. Oncol. 31(29), 3639-3646 (2013).

6. Dumke AK, Pelz T, Vordermark D. Long-term results of radiotherapy in anaplastic thyroid cancer. Radiat. Oncol. 9(1), 90 (2014).

7. Nagaiah G, Hossain A, Mooney CJ, Parmentier J, Remick SC. Anaplastic thyroid cancer: a review of epidemiology, pathogenesis, and treatment. J. Oncol. 2011, 542358 (2011).

8. Sugitani I, Miyauchi A, Sugino K, Okamoto T, Yoshida A, Suzuki S. Prognostic factors and treatment outcomes for anaplastic thyroid carcinoma: ATC Research Consortium of Japan cohort study of 677 patients. World J. Surg. 36(6), 1247-1254 (2012).

9. Matsui J, Funahashi Y, Uenaka T, Watanabe T, Tsuruoka A, Asada M. Multi-kinase inhibitor E7080 suppresses lymph node and lung metastases of human mammary breast tumor MDA-MB-231 via inhibition of vascular endothelial growth factor-receptor (VEGF-R) 2 and VEGF-R3 kinase. Clin. Cancer Res. 14(17), 5459-5465 (2008).

10. Matsui J, Yamamoto Y, Funahashi Y et al. E7080, a novel inhibitor that targets multiple kinases, has potent antitumor activities against stem cell factor producing human small cell lung cancer H146, based on angiogenesis inhibition. Int. J. Cancer 122(3), 664-671 (2008).

11. Okamoto K, Kodama K, Takase K et al. Antitumor activities of the targeted multi-tyrosine kinase inhibitor lenvatinib (E7080) against RET gene fusion-driven tumor models. Cancer Lett. 340(1), 97-103 (2013). 
12. Schlumberger M, Tahara M, Wirth LJ et al. Lenvatinib versus placebo in radioiodine-refractory thyroid cancer. N. Engl. J. Med. 372(7), 621-630 (2015).

-• Pivotal clinical trial and first demonstration of lenvatinib efficacy in a Phase III, randomized study.

13. Tahara M, Schlumberger M, Elisei R et al. Exploratory analysis of biomarkers associated with clinical outcomes from the study of lenvatinib in differentiated cancer of the thyroid. Eur. J. Cancer 75, 213-221 (2017).

- Analyses of tumor and serum biomarkers and their associations with efficacy outcomes from the Phase III study of lenvatinib in radioiodine-refractory differentiated thyroid cancer.

14. Bruix J, Cheng AL, Meinhardt G, Nakajima K, De Sanctis Y, Llovet J. Prognostic factors and predictors of sorafenib benefit in patients with hepatocellular carcinoma: analysis of two Phase III studies. J. Hepatol. 67(5), 999-1008 (2017).

15. Jin H, Sun J, Zhu K et al. The prognostic value of neutrophil-lymphocyte ratio is superior to derived neutrophil-lymphocyte ratio in advanced gastric cancer treated with preoperative chemotherapy and sequential R0 resection: a 5-year follow-up. Onco. Targets Ther. 10, 2655-2664 (2017).

16. Luo G, Liu C, Cheng H et al. Neutrophil-lymphocyte ratio predicts survival in pancreatic neuroendocrine tumors. Oncol. Lett. 13(4), 2454-2458 (2017).

- Study demonstrating that neutrophil-lymphocyte ratio was an independent predictor of overall survival in patients with pancreatic neuroendocrine tumors.

17. Tahara M, Kiyota N, Yamazaki T et al. Lenvatinib for anaplastic thyroid cancer. Front. Oncol. 7, 25 (2017).

- A Phase II, single-arm, open-label study of lenvatinib in patients with thyroid cancer, including anaplastic thyroid cancer.

18. Kiyota N, Schlumberger M, Muro K et al. Subgroup analysis of Japanese patients in a Phase III study of lenvatinib in radioiodine-refractory differentiated thyroid cancer. Cancer Sci. 106(12), 1714-1721 (2015).

19. Schlumberger M, Jarzab B, Cabanillas ME et al. A Phase II trial of the multitargeted tyrosine kinase inhibitor lenvatinib (E7080) in advanced medullary thyroid cancer. Clin. Cancer Res. 22(1), 44-53 (2016).

- A Phase II study of lenvatinib in patients with advanced medullary thyroid cancer and an investigation of potential serum biomarkers associated with anti-tumor activity.

20. Cabanillas ME, Schlumberger M, Jarzab B et al. A Phase II trial of lenvatinib (E7080) in advanced, progressive, radioiodine-refractory, differentiated thyroid cancer: a clinical outcomes and biomarker assessment. Cancer 121(16), 2749-2756 (2015).

21. Keizer RJ, Gupta A, Mac Gillavry MR et al. A model of hypertension and proteinuria in cancer patients treated with the anti-angiogenic drug E7080. J. Pharmacokinet. Pharmacodyn. 37(4), 347-363 (2010).

22. Shen CT, Qiu ZL, Luo QY. Sorafenib in the treatment of radioiodine-refractory differentiated thyroid cancer: a meta-analysis. Endocr. Relat. Cancer 21(2), 253-261 (2014).

23. Kiyota N, Robinson B, Shah M et al. Defining radioiodine-refractory differentiated thyroid cancer: efficacy and safety of lenvatinib by radioiodine-refractory criteria in the SELECT trial. Thyroid 27(9), 1135-1141 (2017).

24. Ito Y, Onoda N, Ito KI et al. Sorafenib in Japanese patients with locally advanced or metastatic medullary thyroid carcinoma and anaplastic thyroid carcinoma. Thyroid 27(9), 1142-1148 (2017).

25. Liu JF, Ba L, Lv H et al. Association between neutrophil-to-lymphocyte ratio and differentiated thyroid cancer: a meta-analysis. Sci. Rep. 6, 38551 (2016).

26. Manatakis DK, Tseleni-Balafouta S, Balalis D et al. Association of baseline neutrophil-to-lymphocyte ratio with clinicopathological characteristics of papillary thyroid carcinoma. Int. J. Endocrinol. 2017, 8471235 (2017).

27. Demir Y, Úçler R, Sürücü E, Turan M, Balli Z, Şengöz T. Temporary changes in neutrophil-to-lymphocyte, platelet-to-lymphocyte ratios, and mean platelet volume reflecting the inflammatory process after radioiodine therapy. Nucl. Med. Commun. 37(4), 393-398 (2016).

28. Galdiero MR, Varricchi G, Marone G. The immune network in thyroid cancer. Oncoimmunology 5(6), e1168556 (2016).

29. Cho JS, Park MH, Ryu YJ, Yoon JH. The neutrophil to lymphocyte ratio can discriminate anaplastic thyroid cancer against poorly or well differentiated cancer. Ann. Surg. Treat. Res. 88(4), 187-192 (2015).

30. Haugen BR, Alexander EK, Bible KC et al. 2015 American Thyroid Association management guidelines for adult patients with thyroid nodules and differentiated thyroid cancer: the American Thyroid Association Guidelines Task Force on Thyroid Nodules and Differentiated Thyroid Cancer. Thyroid 26(1), 1-133 (2016). 